\title{
Fluorescent Staining of Silicone Micro-and Nano-patterns for Their Optical Imaging
}

\author{
H.Samet Varol ${ }^{\mathrm{a}, \mathrm{b}}$, Stefan Seeger ${ }^{\mathrm{a},{ }^{*}}$ \\ ${ }^{a}$ Department of Chemistry, Universität Zürich, Zürich, CH 8057 \\ ${ }^{b}$ Ernst-Berl Institut für Technische und Makromolekulare Chemie, Technische Universität \\ Darmstadt, Alarich-Weiss-Str. 12, Darmstadt, D-64287
}

\begin{abstract}
Performance of engineered surfaces can be enhanced by making them hydrophobic or superhydrophobic via coating them with low-surface-energy micro-and nano-patterns. However, the wetting phenomena of particularly irregular shape and spacing (super)hydrophobic patterns such as polysiloxane coatings are not yet fully understood from a microscopic perspective. Here, we show a new method to collect 3D confocal images from irregular polysiloxane micro-and nanorods from a single rod resolution to discuss their wetting response over long liquid/solid interaction times and quantify the length and diameter of these rods. To collect such 3D confocal images, fluorescent dye containing water droplets were left on our superhydrophobic and hydrophobic polysiloxane coated surfaces. Then their liquid/solid interfaces were imaged at different staining scenarios: (i) using different fluorescent dyes, (ii) when the droplets were in contact with surfaces, or (iii) after the droplets were taken away from the surface at the end of staining. Using such staining strategies, we could resolve the micro-and nanorods from root to top and determine their length and diameter, which were then found to be in good agreement with those obtained from their electron microscopy images. 3D confocal images in this paper, for the first time, present the long-time existence of more than one wetting state under the same droplet in contact with surfaces, as well as external and internal three-phase contact lines shifting and pinning. In the end, these findings were used to explain the time-dependent wetting kinetics of our surfaces. We believe that the proposed imaging strategy here will, in the future, be used to study many other irregular patterned (super)antiwetting surfaces to describe their wetting theory, which is today impossible due to the complicated surface geometry of these irregular patterns.
\end{abstract}

\section{Corresponding author(s)}

*Stefan Seeger (sseeger@chem.uzh.ch) 


\section{Introduction}

Nature has countless examples of tailoring interactions between liquids and surfaces, and these examples have inspired scientists to formulate functional (super)antiwetting surfaces for more than 200 years. ${ }^{[1]}$ Among different nature-inspired surfaces, great attention is given to the chemical and geometrical enhancement of the surfaces in order to make them hydrophobic (contact angle, $\theta_{C A}>90$ ) and superhydrophobic, $\theta_{C A}>150$. Chemical modifications of smooth surfaces can merely reach about $120^{\circ}$ to make them hydrophobic. ${ }^{[2,3]}$ To amplify hydrophobicity and obtain superhydrophobicity, it is necessary to increase the roughness factor by creating micro or nano-patterns. ${ }^{[4]}$ Superhydrophobicity (in general, superantiwetting) is only a factor in the survival of the trapped air (plastrons) layers between liquid and micro-and nano-patterns of the solid layer. ${ }^{[5,6]}$ Transition from the non-wet state where these plastrons exist (Cassie-Baxter state) to the wetting state with the absence of these air layers (Wenzel state) ends with the loss of the superantiwetting properties. Therefore, while formulating superhydrophobic surfaces with high stability, it is essential to understand the fundamental reason behind the wetting transitions, such as time-dependent imaging of Cassie to Wenzel state transition $(\mathrm{C} \rightarrow \mathrm{W})$ at a nanoscale.

In general, hydrophobic and superhydrophobic surfaces have many surface inhomogeneities and distinctive intrinsic roughness features rather than being perfectly smooth. ${ }^{[3,7]}$ Such imperfections of surfaces create variations in $\theta_{C A}$ due to irregularities in (i) lateral and horizontal droplet (de)pinning, (ii) three-phase (water-solid-air) contact lines at the droplet periphery, ${ }^{[7]}$ and (iii) solid-liquid contact area. ${ }^{[7]}$ The whole picture of $\mathrm{C} \rightarrow \mathrm{W}$ transitions and $\theta_{C A}$ of superantiwetting surfaces must be studied by considering such variations with the surface chemistry and roughness parameters. The most used approach to study surface imperfection effect on different wetting transitions is to optically resolve the interface where liquid interacts with individual micro and nano-features of the surfaces. To date, the interface between a sessile water droplet and (super)hydrophobic micron-sized pillars has been mainly visualized by laser scanning confocal microscopy, ${ }^{[8,9]}$ environmental scanning electron microscopy $(\mathrm{ESEM})^{[10]}$, and reflection interference contrast microscopy (RICM). ${ }^{[1]}$ RICM is a potent tool to live-image details of a droplet on top of a micropillar surface with a high temporal and spatial (submicron) resolution in 2D (horizontal). However, RICM cannot provide a 3D visualization of surfaces. The third dimension (vertical) is crucial to understand the transition from one wetting state to another. ${ }^{[12]}$ ESEM could resolve the liquid-solid interface with a very high spatial resolution in 
$3 \mathrm{D}$; however, its temporal resolution is limited. ${ }^{[11]}$ Confocal imaging is the most convenient method for 3D visualization of drop-air and drop-substrate interfaces with a better temporal resolution than ESEM. ${ }^{[12]}$ Nevertheless, these studies are limited to discussing the antiwetting of identical shape and micron-sized pillars with precise surface-to-surface spacing since their mathematical description is easier to explain the surface wetting. However, similar to natural examples such as lotus leaf, many engineered superantiwetting coatings have irregular hierarchical structures, and microscopic methods to visualize the interface of such irregular structures and liquids are limited. ${ }^{[13]}$ A critical example for irregular size and shape (super)hydrophobic coatings is micro-and nano- polysiloxane (or silicone) coatings. ${ }^{[4,13]}$ Since 2003, polysiloxane coating has received significant attention among other superantiwetting coating technologies, thanks to their chemical and environmental stability on the coated surfaces. ${ }^{[5,14-16]}$ The growth of silicone patterns is possible in various morphologies such as rods, nanofilaments (known as silicone nanofilaments, SNFs), star, and volcano shapes. ${ }^{[17]}$ Among them, SNFs are the most known and widely used superhydrophobic silicone coating. ${ }^{[17]}$ One very recent use of SNF coating is to build two-level superhydrophobic surfaces via growing SNFs around lithographically fabricated micropillars. ${ }^{[18]}$ In such surfaces, authors could successfully reverse the Cassie to Wenzel state thanks to plastrons' presence, keeping the surface fully dry even if the air layer between micron-sized pillars does not exist anymore. Even though polysiloxane coatings are getting more and more popular, to the best of our knowledge, to date, no confocal imaging has not been performed to visualize the interface between water and these polysiloxane patterns. Confocal imaging of the liquid/solid interface from a single polysiloxane pattern (e.g., rod) resolution could be helpful to describe the theory of wetting of the polysiloxane coated surfaces, and findings could also be applied to other irregular hierarchically textured antiwetting surfaces. In previous confocal studies of the surfaces with ideal (regular) pillars, fluorescent dyes are generally used to label water and surface micropatterns independently before imaging. However, less interest has been given to use droplets with dyes for alternative applications such as staining of micro-and nano-patterns to quantify their size. In solid surface staining applications by using a fluorescent dye-containing droplet, a significant focus has been given to leave ring-like fluorescent stains on the surfaces upon evaporation of sessile droplets (with the dye inside) known as the coffee ring effect. ${ }^{[19-21]}$ However, no similar fluorescent marking was used to quantify the size and dimension of microand nano patterns in contact with the dye-loaded droplet. This method could be a cheaper and easier alternative to electron microscopy to identify the dimensions of the micro-and nanostructures of (super)hydrophobic coated surfaces and study their wetting transition dynamics. 
Here, we present a new fluorescent staining method to visualize the root-to-top details of polysiloxane micro and nanorods in contact with a sessile droplet. We used this method to image (i) hydrophobic polysiloxane coating with micro-and nanorods and (ii) superhydrophobic polysiloxane coating with two-level topography: micro-rods surrounded by nanofilaments at the base. The interface between the dye-containing sessile droplets and polysiloxane patterns was visualized by inverted confocal laser scanning microscopy as a factor of changing liquid/solid interaction time and using different kinds of fluorescent dyes. Our 3D confocal images showed us that visualizing the polysiloxane micro-and nanorods from a single rod resolution is possible by using different dyes during the droplet is in contact with the surface or even after it is taken away (post-staining). Presented 2D and 3D confocal images demonstrate the axial and lateral (de)pinning, type of wetting state at a particular liquid/solid interaction period, the presence of more than one wetting state at a time, and internal and external threephase contact line shifts. These images are helpful to understand the factors playing a direct role in the surfaces' time-dependent wetting kinetics. As an alternative and facile microscopic method, in the end, we analyzed the length and diameter of the micro-and nanorods visible in our 3D images, and we found out that the results are in good agreement with those generated from electron microscopy micrographs.

\section{Results and Discussion}

\section{Growth of polysiloxane micro-and nano-patterns.}

In this work, two polysiloxane (also known as silicone) coated surfaces were studied (Figure 1). These two surfaces consist of different morphology and topography (as single or two-level) of silicone micro-and nano-structures; thus, having different surface antiwetting properties (Figure 2). Polysiloxane coatings were done through the droplet-assisted growth and shaping $(D A G S)$ method $^{[17]}$ in a home-built chemical vapor deposition $(C V D)$ setup at room temperature (RT). To change the morphology of the coatings, we varied the relative humidity (RH, the mixture of wet $\mathrm{N}_{2}$ and dry $\mathrm{N}_{2}$ ), amount of silane precursor (trichloroethylsilane, TCES), and the number of glass slides to coat (see Methods for details). ${ }^{[17]}$ The basic principle of forming polysiloxane micro-and nanorods through the DAGS method is as follows: ${ }^{[17]}$ in a humid environment, the surface of the glass substrates is covered by a thin but non-homogeneous water layer initiating the formation of micro-and nanosized water droplets. These micro-and nano water droplets are thermodynamically stable and act as the "reaction vessels" for polysiloxane 
polymerization. At a stable $\mathrm{RH}$, a reaction is initiated after adding the volatile TCES in the CVD chamber. Evaporated TCES first reacts with the "reaction vessels" and produces monoand polysilanols and hydrochloric acid $(\mathrm{HCl})$ as a by-product. In the end, hydrolysis and condensation reaction occur in these water droplets and initiate the production of silanol and siloxanol species yielding polysiloxane. As reported before, to obtain micro- and nanorod patterning, it is needed to use high humidity conditions that are between $60 \%-80 \% \mathrm{RH}^{\left[{ }^{[17]}\right.}$ In general, at such high RH values and when the reaction conditions are too humid (wet), the water droplets on the surface get too big, and this results typically in the formation silicone volcano (Figure 1b-left) acting as the starting point where the presented rods in this study grow.

As presented in Figure 1 - left, one of our coatings resulted in two-level topography comprising conic shape micro rods and thin (isotropic) silicone-nanofilaments (SNF) at the base. This coating is also called "superhydrophobic surface" due to its surface properties explained later. To obtain such a two-level topographic coating, we first stabilized the "reaction vessels" droplets on the surface of 30 glass slides at $65 \% \mathrm{RH}$. With such a moderately high humidity, we could obtain both large and small water droplets where volcano-shaped silicone and silicone nanofilaments start polymerize, respectively. After we stabilized the humidity in our system, we added $1.5 \mathrm{~mL}$ of TCES inside the reaction chamber (desiccator) to initiate the polymerization. The high amount of volatile silane is essential for the rod growth on the top of each volcano and a uniform layer of SNFs covering the entire substrate base (Figure 1b-inset). As the result of our second coating (Figure 1 - right), we obtained tall and short silicone rods together on the same surface (Figure 1a-b - right). The coating of these rods will be called in the text as "hydrophobic surface" due to its surface response (Figure 2). In a typical DAGS method, before adding the silane into the CVD chamber, the flow of $\mathrm{N}_{2}$ is typically stopped. However, for growing the micro rods presented in Figure 1c-right, we kept the $\mathrm{N}_{2}$ flow running for the first time even after the silane precursor addition during the entire polymerization reaction. Briefly, to obtain such chimney-like coatings, $392 \mu \mathrm{L}$ of TCES was added after $1 \mathrm{~h}$ of stabilization of 5 glass slides at $75 \% \mathrm{RH}$, and the reaction took $16 \mathrm{~h}$ under the constant flush of $\mathrm{N}_{2}$. Like the two-level topographic coatings, our hydrophobic coatings are uniform along the glass slides' entire surface (Figure 1a - right). As explained before, the conical cavity at the tip of the polysiloxane rods has been the indicator of the droplet volume during rod growth. ${ }^{[17,22]}$ As shown in Figure 1-right, the diameter of the cavity at the tip and the diameter of the hydrophobic rods are almost identical. Such biased stability of RH during the polymerization helped the "reaction vessel" water droplets keep their diameter (size) till the end of the reaction 
and helped generate tubular shape rods. However, when we used the typical DAGS method for our superhydrophobic coatings where RH drops over time, we obtained conical-shaped rods (Figure 1a-left) due to decreasing reaction vessel size during polymerization.

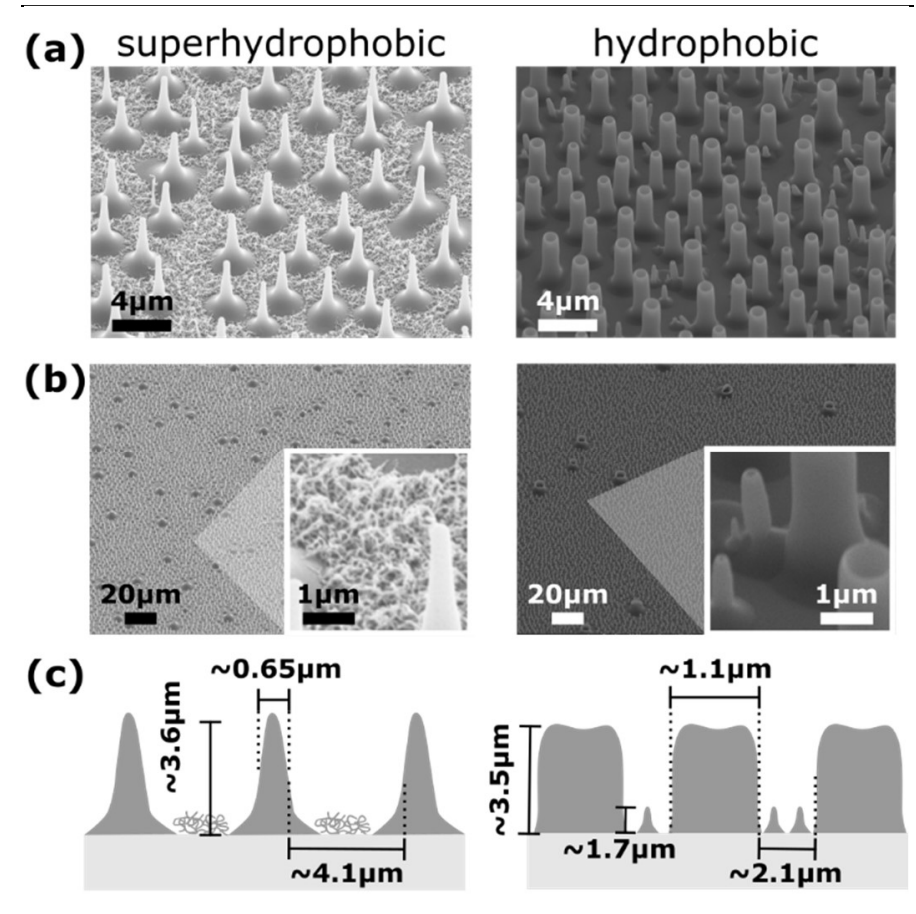

Figure 1. Microstructures of the (left) superhydrophobic and (b) hydrophobic silicone coated surfaces presented with (a) high, (b) low, and (inset) very high magnification SEM images. Insets in (b) present the (left) SNF and a rod from superhydrophobic and (right) small and standard rods of hydrophobic coatings. (c) Essential dimensions of the synthesized polysiloxane rods on surfaces are presented in their illustrations.

The average length $(l)$, diameter $(d)$, and surface-to-surface separation $(S t S)$ of the silicone rods in our samples were calculated from their scanning electron microscopy (SEM) micrographs (see Methods). As illustrated in Figure 1c, the rods' diameters were measured at half of their height. In our two-level topographic silicone coating, the conic shape micro rods have $l \pm \mathrm{sd}=3.6 \pm 0.5 \mu \mathrm{m}, d \pm \mathrm{sd}=0.65 \pm 0.12 \mu \mathrm{m}$, and $S t S \pm \mathrm{sd}=4.1 \pm 1.15 \mu \mathrm{m}$. In our hydrophobic silicone coated surface, the presence of long (standard) and short rods is also observable in the histograms of the length and diameter since they show two maxima (Figure S1b-left). We grouped the collected length and diameter data statistically to generate the average length and diameter values of the rods in our hydrophobic coating. We first fitted a cumulative distribution function (CDF) to length and diameter histograms (Figure S1). Subsequently, data in the 
histograms lying within $0^{\text {th }}$ and $45^{\text {th }}$ frequency of the CDF is used to calculate the $l \pm \mathrm{sd}$ and $d \pm \mathrm{sd}$ of the short rods, and the rest (between $45^{\text {th }}$ and $100^{\text {th }}$ ) is used for finding the $l \pm \mathrm{sd}$ and $d \pm \mathrm{sd}$ of the standard rods (see the red dashed line in Figure S1b). Finally, we found $l \pm \mathrm{sd}=3.5 \pm 0.4 \mu \mathrm{m}$, $d \pm \mathrm{sd}=1.1 \pm 0.2 \mu \mathrm{m}$ and $S t S \pm \mathrm{sd}=2.1 \pm 0.84 \mu \mathrm{m}$ for the standard rods and $l \pm \mathrm{sd} \sim 1.7 \pm 0.5 \mu \mathrm{m}$ and $d \pm \mathrm{sd} 0.4 \pm 0.06 \mu \mathrm{m}$ for the short rods. To verify our length evaluation, we also analyzed the length of standard rods in the SEM micrograph, taken after tilting the stage at $90^{\circ}$ (Figure S2). Length calculated from the SEM images taken at $90^{\circ}$ tilt angle is in good agreement with those reported in Figure1.
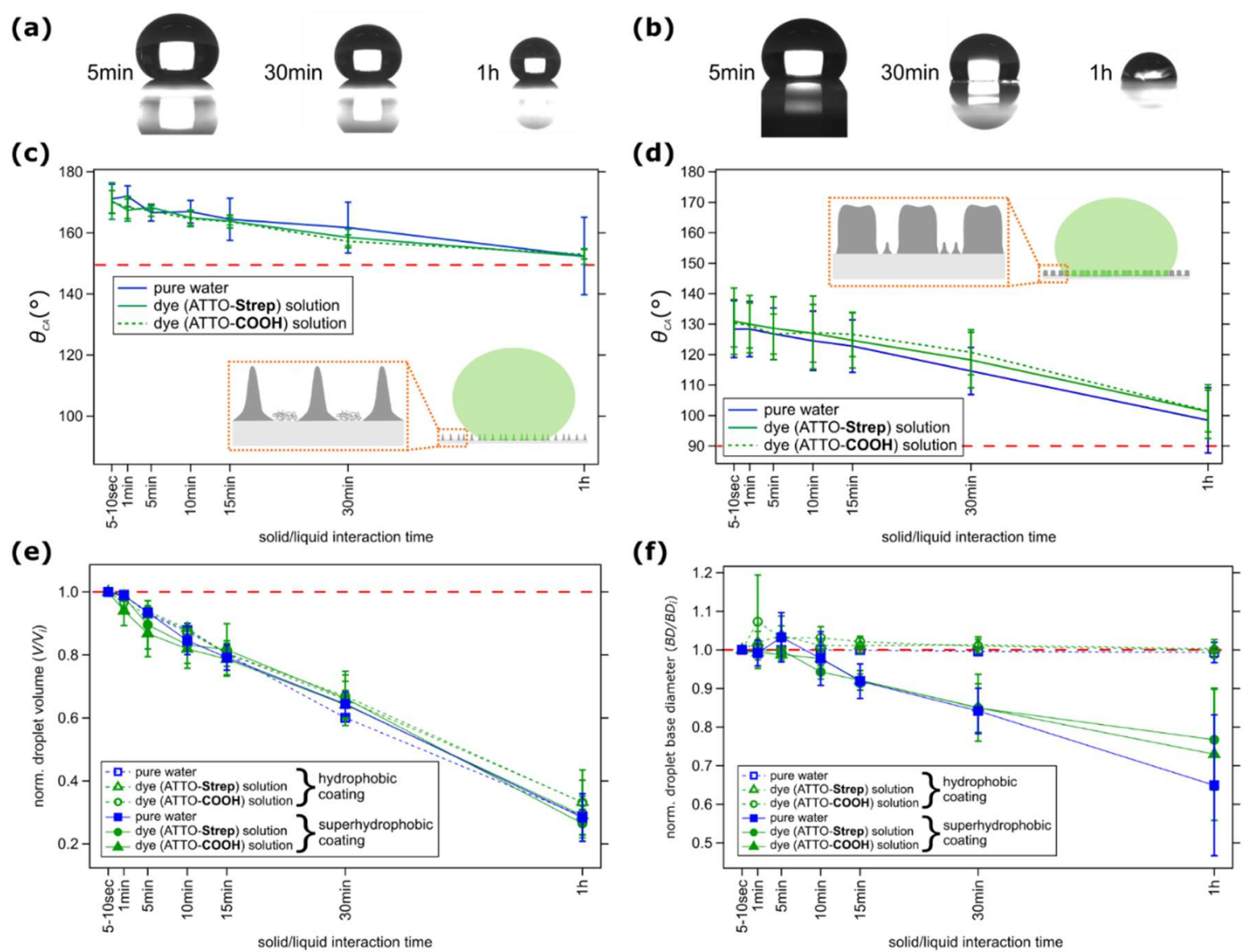

Figure 2. (a-b) Photographic images showing the evaporating $25 \mu \mathrm{L}$ pure water droplets left on (a) superhydrophobic and (b) hydrophobic surfaces. (b-c) $\theta_{C A}$ versus time plots of $25 \mu \mathrm{L}$ of pure water (blue line), Atto-Strep (solid green line), and Atto-COOH (green dashed line) loaded water droplets $(50 \mu \mathrm{g} / \mathrm{mL}$, green) on (b) superhydrophobic and (c) hydrophobic surfaces. Insets illustrate how water droplets sit on both surfaces and the polysiloxane morphologies of the coatings. Red dashed lines in (b) and (c) showing the $\theta_{C A}$ level of $150^{\circ}$ and $90^{\circ}$, respectively. Normalized (e) volume $\left(V / V_{i}\right)$ and (e) base diameter $\left(B D / B D_{i}\right)$ of the $25 \mu \mathrm{L}$ droplets versus time plots. Error bars are SDs from a minimum of 3 different measurements at different locations of a coating. All these measurements were performed in lab conditions $\left(50 \% \mathrm{RH}, 23-24^{\circ} \mathrm{C}\right)$. 


\section{Surface wetting.}

A $25 \mu \mathrm{L}$ of pure or fluorescent dye loaded $(50 \mu \mathrm{g} / \mathrm{mL})$ sessile water droplets were left on the polysiloxane coated surfaces (Figure $2 \mathrm{a}, \mathrm{b}$ ) to measure their apparent contact angle, $\theta_{C A}$ (Figure 2c-d), normalized droplet base diameter, $B D / B D_{i}$ (Figure 2e), and normalized droplet volume, $V / V_{i}$ (Figure 2f) during $1 \mathrm{~h}$ liquid/solid interaction (see Methods). These measurements were done at laboratory conditions $\left(50 \% \mathrm{RH}, 23^{\circ} \mathrm{C}-24^{\circ} \mathrm{C}\right)$. We used two different Atto-633 $\left(\lambda_{f l}=657 \mathrm{~nm}\right)$ type fluorescent dyes, which are conjugated with: (i) Streptavidin protein (called as Atto-Strep, $\left.M_{w} \sim 56 \mathrm{kDa}\right)^{[23]}$ or (ii) acidic carboxyl group (called as $\mathrm{Atto}-\mathrm{COOH}, M_{w}=652 \mathrm{Da}$ ). These dyes are moderately hydrophilic and soluble in water. However, after they are dissolved in water, they might change the sessile droplet's wetting properties, especially during a long time stay on surfaces, for instance, while capturing long integration time 3D confocal images. ${ }^{[11]}$ During such a long time presence of droplets on surfaces, dye concentration in the droplet increases because of water evaporation in time. Therefore, we did our surface wetting tests during $1 \mathrm{~h}$ liquid/solid interaction (Figure 2). We also ensured that all the confocal results presented in this work were collected within a maximum of $1 \mathrm{~h}$ of liquid/solid interaction at identical laboratory conditions $\left(50 \% \mathrm{RH}, 23-24^{\circ} \mathrm{C}\right)$. As predicted, the pure water droplets on both silicone coated surfaces gradually shrank because of water evaporation in ambient laboratory conditions in time (Figure 2a,b). As shown in Figure2a and c, the surface coated with two-level topographic silicone (micro rods and SNF as the base) is superhydrophobic $\left(\theta_{C A}>\right.$ $150^{\circ}$, red dashed line). The sessile droplet left the superhydrophobic surface kept its nearly spherical shape (Figure $2 \mathrm{a}$ ), and $\theta_{C A}$ is higher than $150^{\circ}$ even after $1 \mathrm{~h}$ evaporation (Figure $2 \mathrm{c}$ ). $\theta_{C A}$ does not change with the presence of Atto-Strep and Atto-COOH in water. $\theta_{C A}$ of pure and dye-loaded water droplets are identical even after the droplets get very tiny upon evaporation (Figure 2c). Sliding angle $\theta_{S A}$ of $25 \mu \mathrm{L}$ of droplets with and without dyes is about $\theta_{S A} \sim 2^{\circ}$, and it proves the robust superhydrophobicity $\left(\theta_{S A}<10^{\circ}\right)$ of our two-level coating as well as the absence of dye effect on the sliding angle response too (Figure S3c). Wetting tests of the second polysiloxane coating consists of large and small rods showed the surface response against water as hydrophobic $\left(150^{\circ}>\theta_{C A}>90^{\circ}\right)$. Like the superhydrophobic coating, the presence of the dyes inside the droplet does not affect $\theta_{C A}$ even after $1 \mathrm{~h}$ of droplet evaporation (Figure $2 \mathrm{~d}-\mathrm{f}$ ). Timedependent $\theta_{C A}$ of the two surfaces (Figure c,d) shows that between the $\theta_{C A}$ measured after 5- 10 sec, and $1 \mathrm{~h}$ droplet/solid interactions, there are roughly $12 \%$ and $23 \%$ decreases in the $\theta_{C A}$ for the superhydrophobic and hydrophobic surfaces, respectively. The two-level topography and resistance of plastrons are probably the possible reason for better stability of the measured $\theta_{C A}$ 
even after $1 \mathrm{~h}$ droplet stay on the surface. Time-dependent droplet evaporation and its relation with the droplet base diameter $(B D)$ is essential to understand the performance of the surfaces, such as their stability against wetting as a factor of time. ${ }^{[4,24]}$ Therefore, we checked the changes in droplet volume (Figure 2e) and droplet base diameter (in contact with solid) (Figure 2f) of the droplets with and without dyes as a factor of $1 \mathrm{~h}$ liquid/solid interaction time.

As shown in Figure 2e, the normalized volume of the $25 \mathrm{~mL}$ droplets, $V / V_{i}$ gets linearly smaller in time, independent of the water repellency properties of the surface, the presence, and the type of dyes in water (see Methods for $V / V_{i}$ calculation). Unlikely to $V / V_{i}$, normalized droplet base diameter $\left(B D / B D_{i}\right)$ does not change in time for the droplets left on the hydrophobic surface, but it gets gradually smaller for the droplets on the superhydrophobic surface. Similar to the other surface characterizations in Figure 2, the presence and type of fluorescent dye in water do not play any role in the detected $B D / B D_{i}$ on both surfaces. In conclusion, as presented in Figure 2, adding $50 \mu \mathrm{g} / \mathrm{mL}$ of both ATTO dyes in water does not play any role in the liquid/solid interaction of both superhydrophobic and hydrophobic surfaces used in this study. We also realized that even $1 \mathrm{~h}$ of a water droplet (with or without dyes)/surface interactions, the repellency of both surfaces remains as superhydrophobic $\left(\theta_{C A}>150^{\circ}\right)$ and hydrophobic $\left(\theta_{C A}>90^{\circ}\right)$. It is essential for our confocal images presented here since some of them have been captured at long integration times.

\section{Time-dependent wetting kinetics visualized by $2 D$ snapshot confocal images.}

For microscopic imaging of our polysiloxane-coated surfaces in contact with water, we placed $25 \mathrm{~mL}$ of Atto-Strep or Atto-COOH loaded water droplets $(50 \mu \mathrm{g} / \mathrm{mL})$ on both surfaces and imaged their liquid/solid interfaces by inverted laser scanning confocal microscopy, LSCM (Figure 3a). In our LSCM setup, $d_{\text {lateral }} \sim 300 \mathrm{~nm}$ and $d_{\text {axial }} \sim 650 \mathrm{~nm}$ are the highest possible resolution to achieve by $64 \mathrm{X}$ oil-immersed objective lens $(\mathrm{NA}=1.4)$ at $633 \mathrm{~nm}$ wavelength. In our LSCM setup, we used two different detectors to detect fluorescence emission and the other for detecting the reflection of light from interfaces having significantly different reflective indexes such as the water/air interface (Figure 3a). We used two different fluorescent dye conjugates in this study to understand if the molecular size (or weight) of dyes plays any role in resolving the silicone micro- $\&$ nano-patterns. We first investigate the droplet spreading (in $\mathrm{X}-\mathrm{Y}$ axes) on the hydrophobic (Figure 3c-d) and superhydrophobic (Figure 3e-f) surfaces as a factor of time when droplets with fluorescent dyes are in contact with the surface. We also call 
this spreading of the droplets as external or internal three-phase (water-solid-air) contact line shifting (Figure 3b). 2D (XY) snapshot images in Figure 3 presents the signal from both reflectance (red color) and fluorescent channels (green color). In literature, especially for micron-sized ideal pillar systems, reflection lines from pillar-air and air-water interfaces are used to image the thickness of the air cushion in the Cassie-Bexter state of wetting (Figure 3b- (3)). ${ }^{[8]}$ However, such reflection signals from the two interfaces are detectible only if the size of pillars and, thus, the air cushion between them are large enough to resolve $(>250 \mathrm{~nm}$ in horizontal, $>700 \mathrm{~nm}$ in vertical). In our work, for the first time, we imaged the non-ideal (irregular spacing and size) and significantly small polysiloxane rods compared to the ideal pillars used in the literature. Figure 3c-f presented external and internal three-phase contact lines (Figure 3b) of both surfaces in contact with dye-loaded droplets. Independent of the fluorescent dye or surface repellency of the coatings, shortly (5 min) after placing dye-loaded droplets on both surfaces, we detected a clear appearance of the fluorescent signal (green) appearing inside of reflectance signal (red region). Reflectance signal originates from the solid/air interface of our surfaces. The appearance of this fluorescence signal is simply the result of $\mathrm{C} \rightarrow \mathrm{W}$ transition (Figure 3b-(1)) or Cassie to intermediate transition (Figure 3b-(2)), for instance, Cassie to nano-Cassie transition where plastrons in SNF resist against wetting. In a hydrophobic surface $\left(150^{\circ}>\theta_{C A}>90^{\circ}\right)$, air pockets between the micro rods do not exist, and thus Wenzel state of wetting happens quickly (Figure 3b-(1)). Therefore, we detected a uniform and solid fluorescent area (green) without any red traces in between, originating from the reflection signal (Figure 3c-d). The absence of reflectance signal where the fluorescent signal was detected is simply the reason for having no air-solid and/or air-water interface. We also realized that, even at long liquid/solid interaction times, no green signal spreads in the $\mathrm{XY}$ direction in our hydrophobic surface in both external and internal three-phase lines, and they stay stable (Figure 3c-d). This stability in the fluorescent signal spreading is in good agreement with the data presented in Figure 2e, and it supports the idea of pinning of the droplets. Our method helped us monitoring the existence and stability of the internal and external three-phase lines on our hydrophobic coatings in contact with water, and we believe pinning is responsible for their presence and stability. 


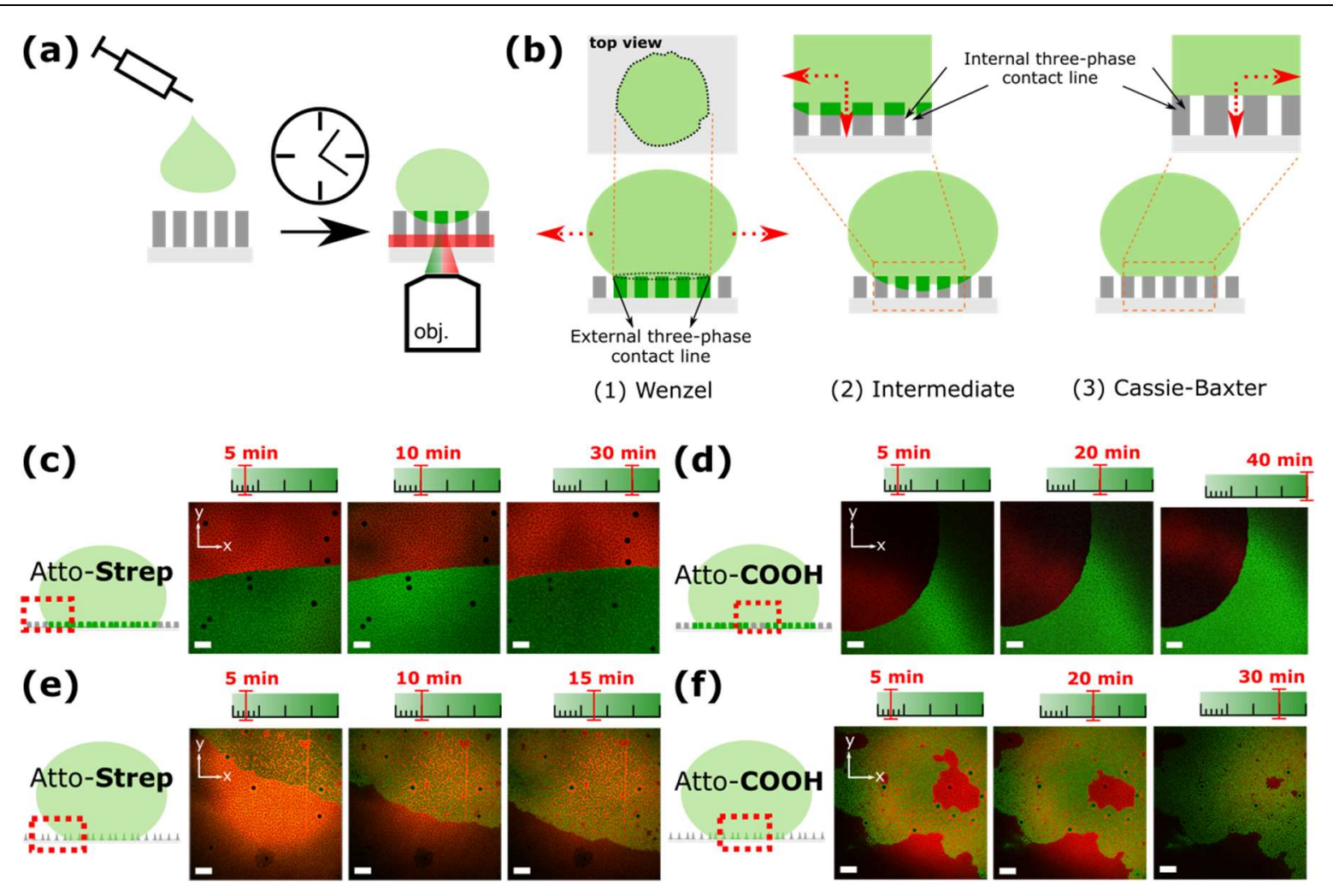

Figure 3. (a) Illustration of our fluorescent staining and subsequent inverted microscopy imaging. The red rectangle close to the substrate represents the reflection emission detected close to the substrates (air-solid interface). Dark green areas on pillars represent fluorescence signals emitted from the dye molecules in the water droplet in contact with the micro-and nano-patterns. (b) Schematic illustration of (1) Wenzel state, (2) intermediate (e.g., nano-Cassie), and (3) Cassie-Baxter wetting states. Black arrows show the position of external and internal three-phase contact lines. The red dotted arrows show the direction of droplet movement (spreading) as a factor of liquid/solid interaction time. Asymmetric droplet shape presented in the top view of the Wenzel state (it is also valid for other wetting states) represents the local corrugation of the external three-phase contact line based on the surface roughness. (c-f) Fluorescent staining by using Atto-Strep (c\&e) and Atto-COOH (d\&f) fluorescent dyes (green channel) in $25 \mu \mathrm{L}$ water droplets sitting on (c-d) hydrophobic and (e-f) superhydrophobic surfaces as a factor of different droplet/surface interaction time (time scales on each image). The red channel is reflectance. The presented 2D images show the (c\&e) external and the (d \&f) internal three-phase contact lines of droplets in contact with the surfaces. Scale bars are $30 \mu \mathrm{m}$.

For the superhydrophobic surface $\left(\theta_{C A}>150^{\circ}\right)$, independent of the fluorescent dye type, the fluorescent signal at the external (Figure 3e) and internal (Figure 3f) three-phase contact lines spreads in larger areas in time (see also Figure S4a, S5). Unlike the hydrophobic surfaces, the fluorescent signal (green) in the superhydrophobic surface is not solid, and it contains red traces (from reflectance signal) in between the green area. The appearance of these red traces is due to plastrons' presence (between non-wet SNFs), where reflectance signal from the air-water and air-solid gets visible in these small traces. In terms of wetting, having such red traces between 
fluorescent signals even at long interaction times (ca. 50min) proves the presence of a nanoCassie wetting state thanks to the plastrons within SNF (Figure S5).

Findings from the snapshot images above provide information about the droplets' timedependent spreading mechanism in 2D (XY axes). However, as illustrated in Figure 3b, to have a complete view of the present wetting states and time-dependent wetting behavior of the surfaces in contact with water droplets, we need 3D visualization of interfaces. Such a 3D view will help us to answer questions where 2D images cannot answer. For instance, in superhydrophobic surfaces, we need 3D confocal images to understand if the reflection only originates from the plastrons within SNF air-pockets or there is a possibility to collect reflection signals from the interface between air-micro rods (Cassie-Baxter state) also exists. Also, 3D imaging at a single rod resolution is needed in the hydrophobic surface to understand how unusual micro-dry regions under droplet base area (Figure $3 \mathrm{~d}$ ) survive even long after the $\mathrm{C} \rightarrow \mathrm{W}$ transition. Unlikely the many water-repellent ideal pillar surfaces in literature, our polysiloxane rods have non-ideal shape (variations in their size as short and tall rods in hydrophobic surface), the non-uniform distance between them, and some large irregular morphologies (large volcano shape structure in the superhydrophobic coating in Figure 1b). 3D profiles will also help us checking for the first time the effect of having such non-ideal (super) water repellent patterns and their effect on forming the asymmetric droplet three-phase contact line as illustrated in Figure 3b-left. In general, surface irregularities and defects represent local corrugation of the external three-phase contact line, and this can be observable in different wetting states as well as Wenzel state, and this corrugation has a direct effect on the time-dependent changes in $\theta_{C A}{ }^{[7,25,26]}$ Therefore, below, we will investigate the surfaces in contact with dye-loaded droplets in $3 \mathrm{D}$ to investigate time-dependent wetting kinetics of the surfaces and use these images to calculate the size of the micro-and nano polysiloxane rods. 
(a)
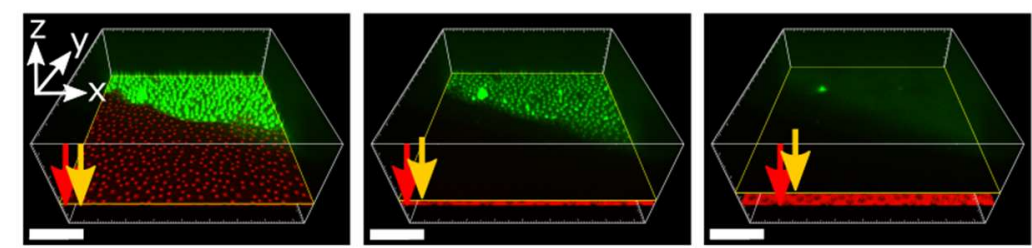

\section{Atto-Strep}
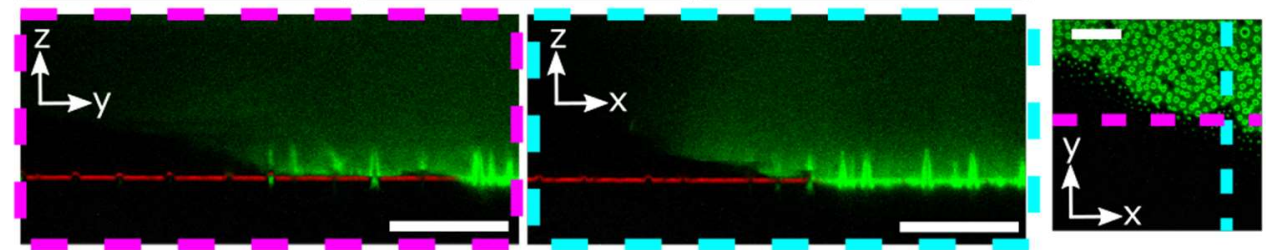

(b)
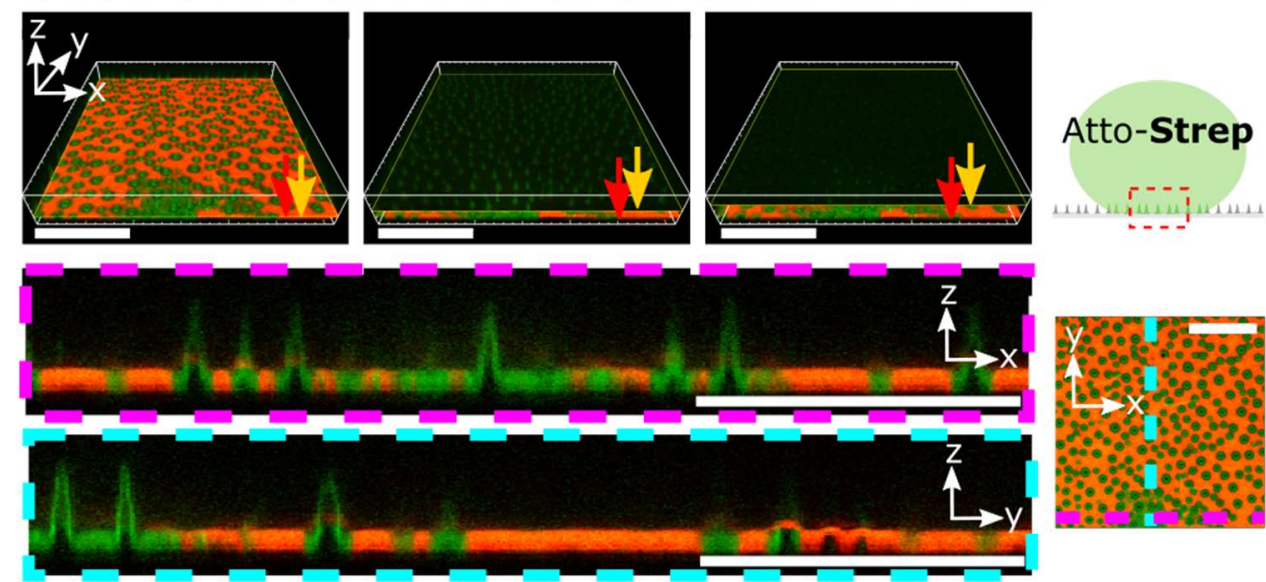

(c)
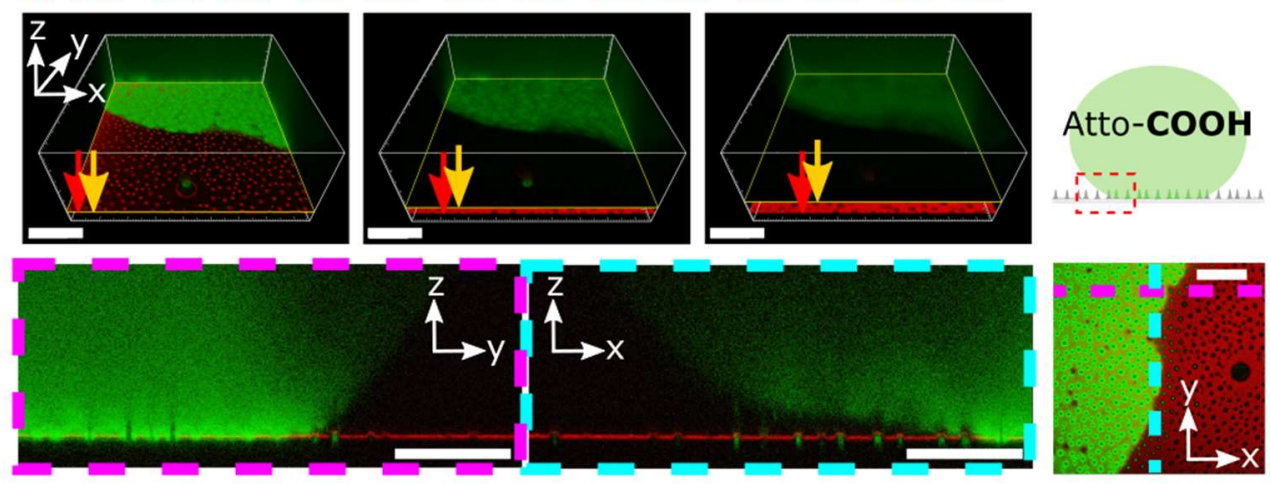

(d)
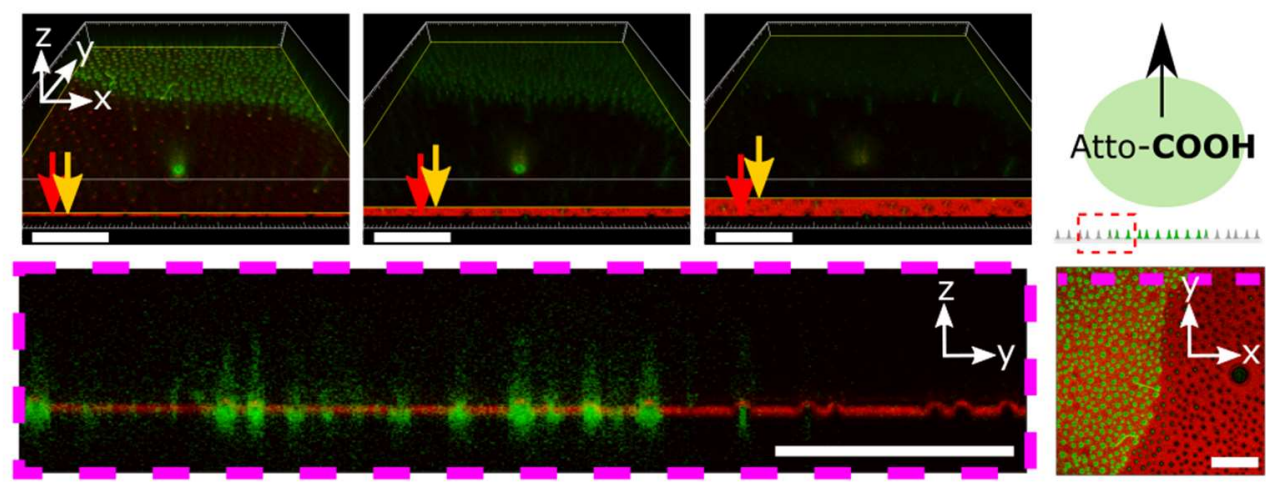

Figure 4. 3D Z-stack confocal images (fluorescence signal: green and reflectance signal: red) of the superhydrophobic patterns carrying $25 \mu \mathrm{L}$ droplets of (a-b) Atto-Strep and (c-d) Atto-COOH dye solution $(50 \mu \mathrm{g} / \mathrm{mL})$ on the top. (a-d-left) XY-Orthoslicers presented in 3D stack images are placed roughly close to the bottom (top-left), middle (top-right), and highest (bottom-left) points of the rods. Red and orange color arrows show the level of substrate surface and the XY-orthoslicer in the z-axes, respectively. Presented 3D images in (a), (b), 
and (c) were taken after $55 \mathrm{~min}, 40 \mathrm{~min}$, and $55 \mathrm{~min}$ liquid/solid interaction periods, respectively. 3D confocal image in (d) shows the stained superhydrophobic rods after the dye-containing droplets were taken away at the end of the $55 \mathrm{~min}$ staining. Images were taken at (a, c \&d) the external three-phase contact line and (b) at the center at the droplet base. The location where the 3D stack images were taken for (c) and (d) is identical. (bottom (a-d)) $\mathrm{XZ}$ - and YZ-Section-slicers which were taken from their 3D images, are presented. The exact positions where the XZ-, YZ-Section-slicers were taken are also shown in the XYOrthoslier images (below illustrations); these positions were indicated by using identical pink and cyan dashed lines. Scale bars (white bars) are $30 \mu \mathrm{m}$.

\section{Studying the wetting transitions of the surfaces in $3 D$.}

\section{Superhydrophobic (two-level topographic) surface:}

3D z-stack images of $25 \mu \mathrm{L}$ dye-loaded droplets interacting with superhydrophobic and hydrophobic surfaces are presented in Figure 4 and Figure 5, respectively. Such 3D imaging allows us to resolve silicone micro-and nanorods interacting with the liquid from a single rod resolution in axial (XY) and lateral (Z) dimensions. We first added Atto-Strep loaded droplet $(50 \mu \mathrm{g} / \mathrm{mL})$ on the top of the superhydrophobic surface and waited a minimum of $40 \mathrm{~min}$ before capturing the $3 \mathrm{D}$ images. Afterward, we imaged the liquid/solid interface at the external (Figure 4a) and internal (Figure 4b) three-phase contact lines. 3D z-stack images in Figure 4a- b show that the protein-based dye can make the rods detectable from root to top. When the $\mathrm{XY}$ - orthoslicer position (red and orange arrows in Figure 4) was placed close to the roots of the rods, we detected both reflection (red) and fluorescence (green) signals together (Figure 4a- $d$ - top-left). However, when we shift the position of the XY-Orthoslicers to the middle and the top of the rod height, only fluorescence signal (no reflectance signal) was detectible ((Figure 4a-d - top, Figure S6a). A similar absence of reflectance signal distant from the substrate surface was detected in all the other 3D images in this study (Figure 4, 5 \& S6). Therefore, we could conclude that root-to-top visualization of the superhydrophobic and hydrophobic rods (Figure 5) is possible only by fluorescence imaging but not reflection. Local corrugations of the external three-phase contact line (Figure $3 \mathrm{~b}$ ) are presented in the $\mathrm{XZ}$ and YZ cross-section (XZ- and YZ-orthoslicers) images in the lower panels of Figure $4 \mathrm{a}$ and $\mathrm{b}$. The cross-section images show that even after $55 \mathrm{~min}$ liquid/solid interaction time, some rods at the external three-phase contact line can resist against micro-to-nano Cassie transition and stay stable at the micro-Cassie state of wetting. Therefore, the external three-phase contact line profile gets nonsymmetric (orthoslice image in the blue dashed box in Figure 4a), similar to the illustration in Figure 2b-left. At the same time, interestingly, we detected a strong fluorescence 
signal close to the base (where SNF locates) in the same image that looks like a Wenzel state of wetting. Collecting such a strong signal from the three-phase contact line could be simply the result of the coffee-stain effect, in which dye molecules in the evaporating water droplet (in $55 \mathrm{~min}$ ) tend to gather close to the three-phase line. ${ }^{[27]}$ To check if Wenzel transition is possible in our two-level topographic surfaces, we also collected images from the regions close to the center of the sessile droplet base (Figure $4 b$ ). When we take an image in the center of the base droplet area (Figure 4b), we realized that the ATTO-Strep dye makes only the micro rods visible but not the SNF at the base. After a long liquid/solid interaction time (40min), only a tiny portion of the base SNF structure could be stained. Therefore, we believe a $25 \mu \mathrm{L}$ sessile water droplet on our superhydrophobic surface can have only a micro-to-nano Cassie wetting transition but no nano-Cassie to Wenzel transition. The intense fluorescent signal close to the three-phase line is due to the dye molecules' coffee-stain effect. Besides, after taking the ATTOStrep containing droplet away from the surface, we realized fluorescent emission from only a small portion of the rods close to their roots but not from their entire body or not from SNFs (Figure S7). We believe that no staining of the SNFs is further proof of having a nano-Cassie wetting state. ATTO-COOH loaded droplet was also used to image the superhydrophobic surface's three-phase contact line (Figure 4c). The YZ orthoslice image in Figure 4c (in cyan dashed line box) presents the resistance of the rods against micro-to-nano Cassie transition, as previously shown in Figure 4a. However, unlike the data presented in Figures $4 \mathrm{a}$ and $\mathrm{b}$, the Atto-COOH dye-loaded droplet sitting on the superhydrophobic surface cannot help us resolve the micro rods. Interestingly, we could resolve almost root-to-top Atto-COOH stained microrods only after the droplet was taken away at the end of $55 \mathrm{~min}$ of liquid/solid interaction period (Figure 4d). As reported above, such root-to-top staining is not possible after the ATTO-Strep dye-containing droplet was removed from the surface (Figure S7). The molecular weight difference between the dyes probably explains the difference in resolving rods with different dyes. As a macromolecular fluorophore, ATTO-Strep has a significantly larger (ca. 55kDa ${ }^{[23]}$ compared to ATTO-COOH $(652 \mathrm{~g} / \mathrm{mol})$. When an ATTO-COOH dye-containing water droplet sits on the surface, large amounts of small dye molecules concentrate close to the superhydrophobic surface without spreading to the substrate surface due to the nano-Cassie state of wetting (Figure 3b-(2)). This high number of dye cumulations prevents us from resolving the rods but is almost a solid layer of fluorescence emission (Figure 4c). When we place a droplet including an identical concentration but big-sized ATTO-Strep dye, we have the ideal number of dyes to make the micro-rods entirely resolvable. About staining the rods after the dye-containing droplet is taken away, the presence of many small size ATTO-COOH dyes 
at the droplet/solid interface is advantageous since they remain around the rods even after the droplet is taken away (Figure 4d). However, macromolecular ATTO-Strep dyes prefer to remain mainly in the droplet when the droplet was taken from the surface (Figure S7).

\section{Hydrophobic surface:}

Hydrophobic silicone coated surface was also studied when the surface was in contact with $25 \mu \mathrm{L}$ of sessile water drops loaded with ATTO-Strep (Figure 5a) or ATTO-COOH (Figure 5b-c). Unlike confocal results of the superhydrophobic surface (Figure 4a-b), both fluorescent dyes make the hydrophobic micro and nanorods visible when the dye-containing droplets sit on the surface (Figure 5a-b). We believe lower water repellency in the hydrophobic coating than the superhydrophobic surface (smaller $\theta_{C A}$, Figure 2) plays a direct role in resolving the hydrophobic rods efficiently. Unlike the superhydrophobic surface, the water can easily wet the substrate's base on our hydrophobic surface. Thus, compared to the superhydrophobic surface interface, more ATTO-COOH dyes spread on the substrate surface too. In the end, the higher number of dyes spreading on the substrate surface results in fewer ATTO-COOH molecules around the hydrophobic rods. This less number of dyes helps us resolve the rods in hydrophobic coating (Figure 5b). However, similar to the superhydrophobic rods, after the droplets were taken away, only remaining Atto- $\mathrm{COOH}$ dye around the hydrophobic rods could make them detectable but not ATTO-Strep (Figure 5c). As explained previously, the number of remaining macromolecular ATTO-Strep dyes around the rods is probably not enough to emit enough fluorescence signal to detect the rods (Figure S8). As indicated in Figure 3c-d, at some micro-locations, the hydrophobic surface resists against wetting (Figure 5a,b-c). Unlike the superhydrophobic surfaces (changes in stained areas), these wetting resistant micro-regions do not present any shift in their internal three-phase lines (Figure 3b-(2)) and do not get narrower in time (Figure 5a-b). We also determined that the internal three-phase contact line is stable even more than 30min droplet contact time (Figure S9a). We believe the pinning effect has a direct role in keeping these non-wet areas stable over time. To the best of our knowledge, the huge rods (see Figure 1b-right) among the other polysiloxane rods in our hydrophobic coating are considered as surface defects. Similar to the local deformations creating local corrugations on the external contact line (Figure $3 b),{ }^{[7]}$ these large rods - defects - also inhibit the droplet from reaching the global energy minimum by uniformly wetting the surface. As a result, micro-regions around these large rods remain dry even at long liquid/solid interaction times since these regions create different energy barriers, and the system cannot go to a global-minimum wetting state. The imaging method in this work, 
for the first time, helped us to image such local corrugations both on internal and external threephase contact regions and monitor their long-time stability.
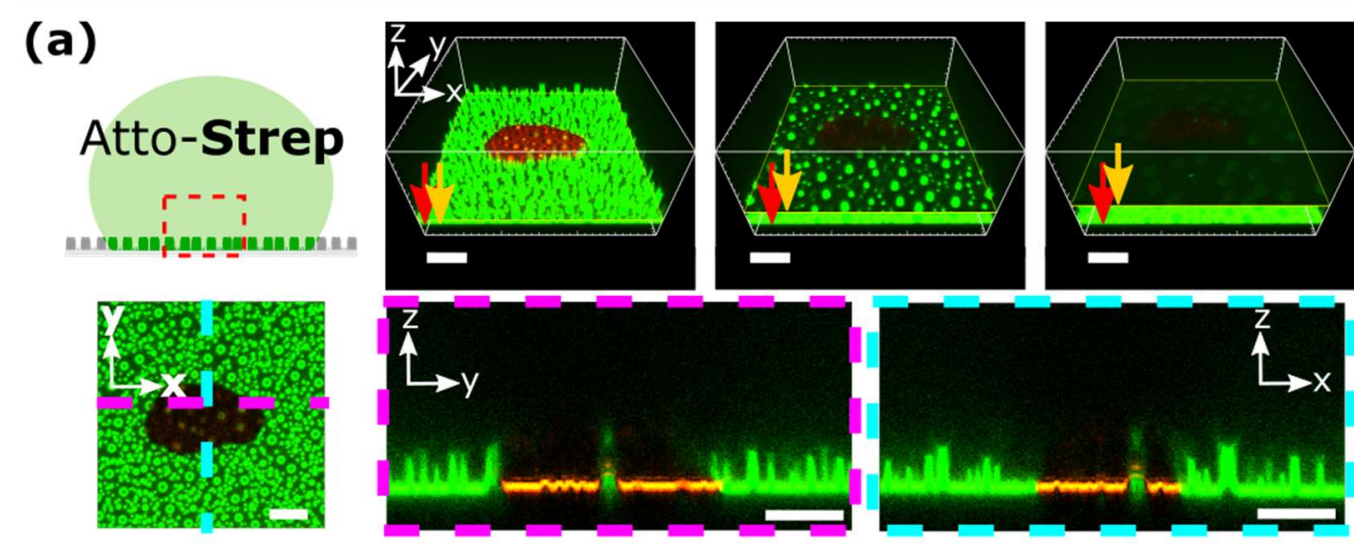

(b)
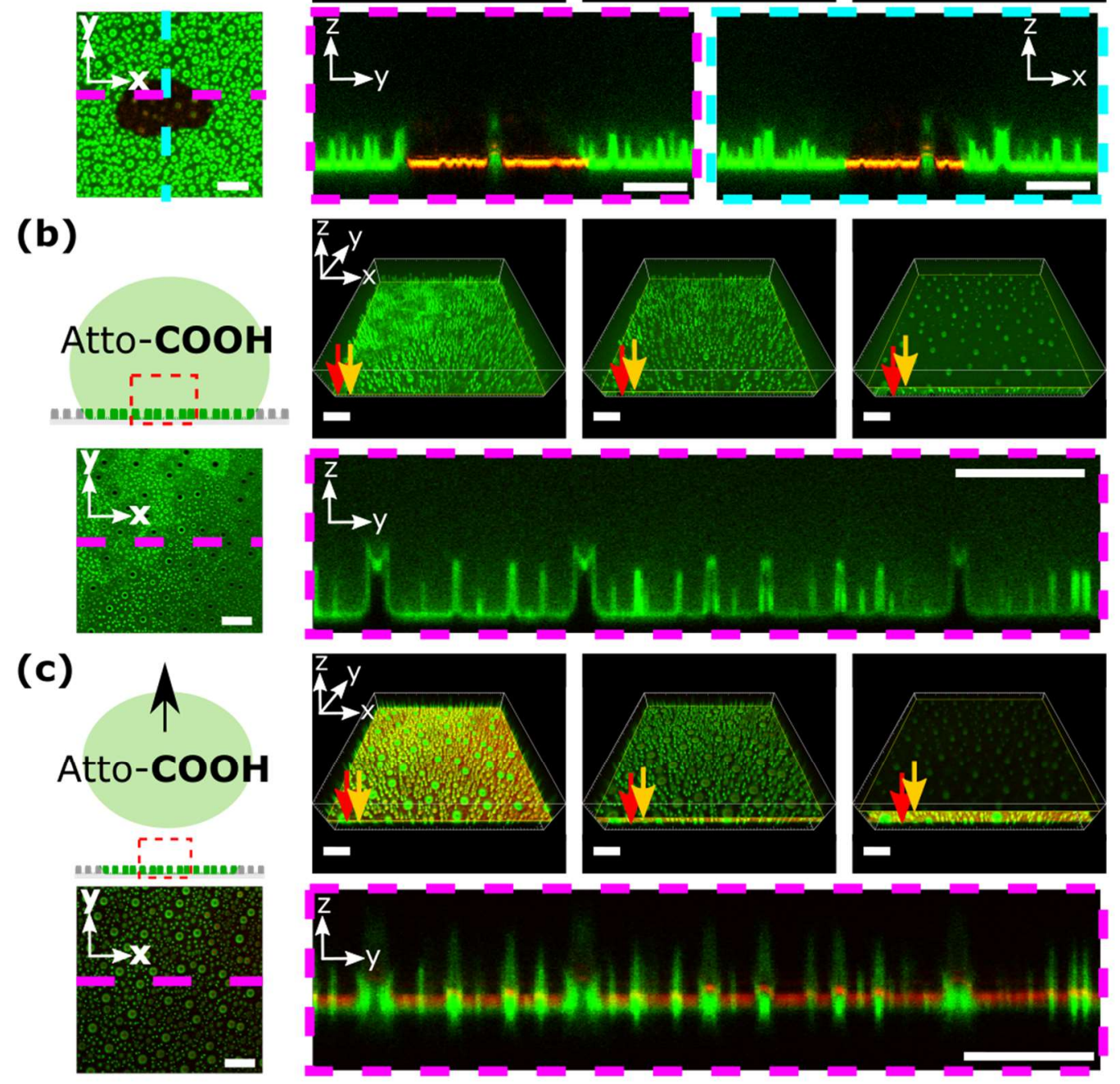

Figure 5. Z-stack confocal images of the hydrophobic surface when $25 \mu \mathrm{L}$ of water droplets with $(50 \mu \mathrm{g} / \mathrm{mL}$ ) (a) Atto-Strep or (b) Atto-COOH dyes contact with the surface for (a) $10 \mathrm{~min}$ and (b) $12 \mathrm{~min}$. Red and green channels belong to the reflection and fluorescence signals, respectively. (c) 3D Z-stack images present the staining of the rods after the Atto-COOH containing droplet was taken away after 30min. (a-c, top-right) XY-Orthoslicers in the 3D Z-stack images were placed close to the root (left), middle (middle), and the top (right) of the imaged standard rods. Red and orange color arrows show substrate surface and $z$-axis level of the XY-orthoslicer. (a-c, bottom) YZ- and XZ-Section-images of their 3D Z-stack images above. $\mathrm{XY}$-section image on the bottom-left indicates where these $\mathrm{YZ}$ or $\mathrm{XZ}$-section images were taken (blue and cyan dashed lines). Scale bars are $10 \mu \mathrm{m}$. 
In this work, the proposed confocal imaging method allows us to 3D-image each polysiloxane micro-and nanorod with several hundred nm axial and lateral resolution. Even if this resolution is way limited compared to those from the electron microscopy methods, such as SEM (several $\mathrm{nm}$ resolution), our method can still quantify the dimensions of the resolved nanorods. Therefore, as a possible application of our imaging method, we quantify the length and the diameter of the stained rods upon their staining (Figure 6).

\section{Determining the diameter and length of the micro and nano-silicone rods from their confocal images.}

Scanning electron microscopy images in Figure 1 provide us precise average diameter $\left(d_{\text {avg }}\right)$ and average length $\left(l_{\text {avg }}\right)$ of the rods from their SEM micrographs. Here, we also quantify the $d_{a v g}$ and $l_{\text {avg }}$ of the silicone micro-and nanorods visible in the $3 \mathrm{D}$ confocal fluorescent channel images by using an image processing routine - called surface creation analysis - in Imaris Pro software (see Experimental Section and Table S1). In the end, we checked the consistency of our $d_{\text {avg }}$ and $l_{\text {avg }}$ results from confocal images by comparing them with those obtained from SEM analysis (Figure 1). First, we selected the best confocal 3D images presenting the microand nanorods' root-to-top morphology in their fluorescence channel signal. Therefore, we ran the surface analysis routine to the following 3D confocal images presented previously: Figure $4 b$, presenting superhydrophobic rods in contact with ATTO-Strep containing droplet (Figure $6 a)$; Figure $5 a$, presenting hydrophobic rods in contact with ATTO-Strep containing droplet (Figure 6b); Figure 5b, presenting hydrophobic rods in contact with ATTO-COOH containing droplet (Figure 6c) and Figure 5c, presenting fluorescently labeled hydrophobic rods after the ATTO-COOH containing droplet was taken away from the surface (Figure 6d). Then, we set the display adjustment of all these 3D images' fluorescent channels to a similar maximum, minimum, and gamma (meaning, an integrated correction function to every voxel intensity of the channel) grey values (Table S1 \& S2). In this way, we provide identical contrast and homogenized signal intensity between the different fluorescent images before applying the surface creation. In subsequent surface-creating wizard steps in ImarisPro, we kept the surface fitting parameters identical (see Methods and Table S2 for details). We finally generated the surface fitting of the rods from the fluorescent channel, as shown in Figure 6a-d (see also Figure S10a,b). 
The color-coded images in Figure 6a-d show the height of superhydrophobic and hydrophobic rods within the limits of $1.5 \mu \mathrm{m}$ (blue color) $-5.9 \mu \mathrm{m}$ (red color) and $0.1 \mu \mathrm{m}$ (blue color) $-6.0 \mu \mathrm{m}$ (red color), respectively. As it is done in their 3D confocal images previously, also in Figure 6a-d, XY-orthoslicers are placed close to the root (left), middle (middle), and the top (right) points of the detected rods (red and orange arrows in Figure 6a-d). After color mapping, almost all the superhydrophobic rods in Figure 6a are presented with similar colors (green) due to their uniform height. However, as presented in their SEM images previously (Figure 1 \& Figure $\mathrm{S} 1 b$ ), also color mapping of the rods from the hydrophobic coating showed clear segregation of rods into two classes based on their height. As shown in Figure 6b-d, after the surface fitting, the short rods appear in blue and green colors, and the rest of the rods - standard rods - are colored mainly yellow and red.

As done for the SEM image analysis of the rods in our hydrophobic coating (Figure 6i), we also fitted a cumulative distribution function (CDF) to the rod height histograms obtained from the confocal image analysis of the images from our hydrophobic surface. This fit helped us to separate height data of the small and large (standard) rods in our hydrophobic coating (Figure S1b-d). Identical to the SEM image analysis, the height of rods detected between the $0^{\text {th }}$ and $45^{\text {th }}$ and $45^{\text {th }}-100^{\text {th }}$ percentile assigned to the short and standard rods, respectively. Since the dimension of SNFs in the superhydrophobic coating is not included in our surface creation results, we used the entire data set in length and diameter histograms (0th - 100th) to calculate the length and diameter micro-rods. To generate statistically consistent size results, we needed to collect many elements to analyze (sample size, N). Therefore, we counted (analyzed) almost 300 rods from the superhydrophobic surface $(\mathrm{N} \sim 300)$ to generate $l_{\text {avg }}$ and $d_{\text {avg. }}$. To be consistent, we tried to analyze the same number of hydrophobic rods. Therefore, instead of running the surface fitting analysis to the entire imaged area, as presented in Figure 6b-d, we did surface fitting analysis to a selected portion of the confocal images (Figure S10 and Video S1). 


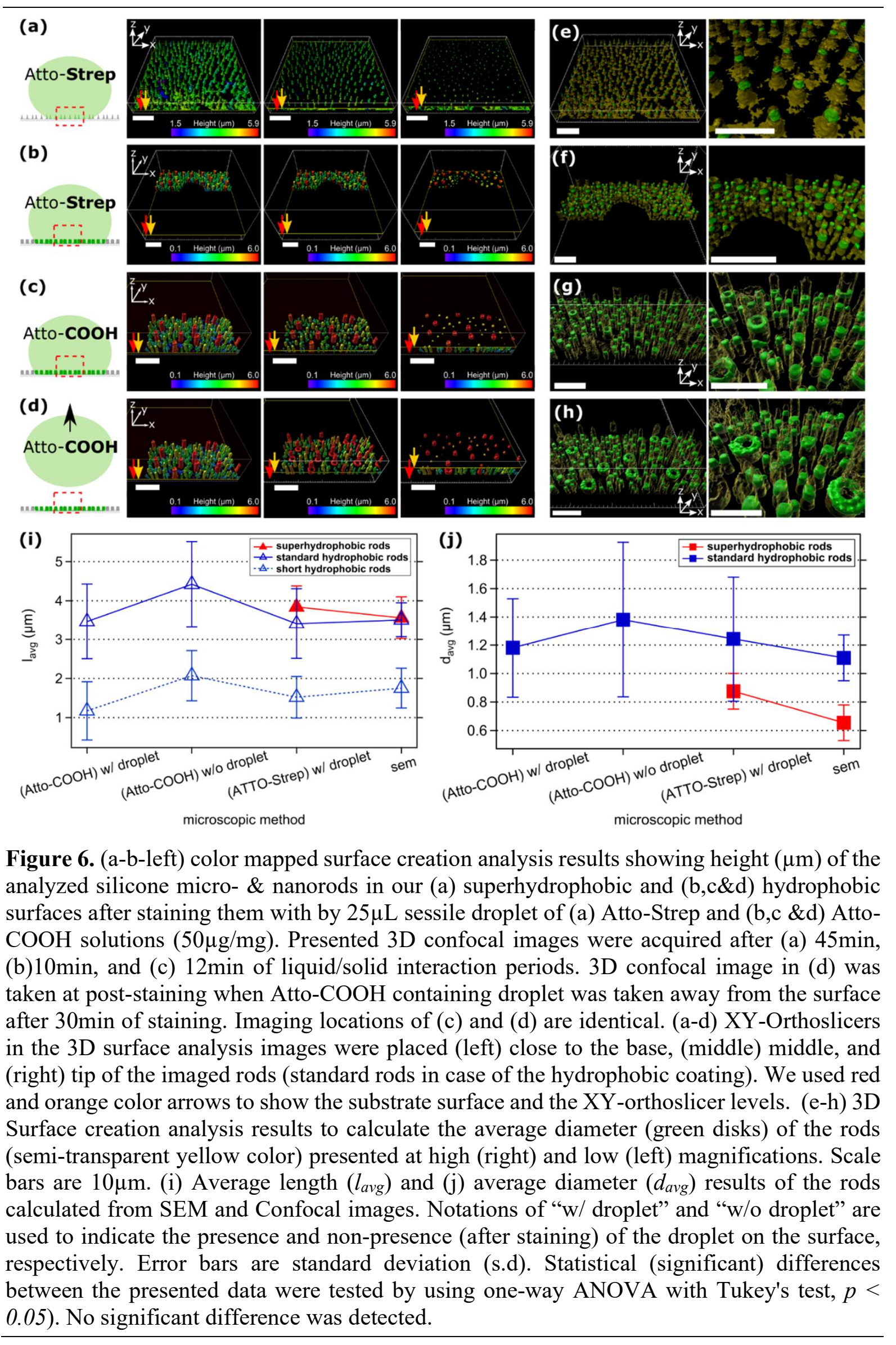


Surface creation analysis in Imaris Pro was also used to quantify the diameter of the rods, $d_{\text {avg }}$ (Figure 6e-h). Identical surface fitting parameters were used to calculate $l_{\text {avg }}$ and $d_{\text {avg }}$ from every 3D confocal image shown in Figure 6a-h. The only difference between the fitting parameters used for $l_{\text {avg }}$ and $d_{\text {avg }}$ is that for the surface fitting of $d_{\text {avg }}$, we limited the surface analysis range (distance) in the Z-direction to a few micrometers instead of running the surface analysis along with the entire height of the rods. After we run surface creation in such a narrow space in Zdirection (see Table S1 for details), the created surfaces result in disk (Figure 6e-f) or donut (Figure 6g-h) shapes, and they locate in the middle height of the rods. The surface analysis results from the diameter (green) and length (semi-transparent yellow) are presented together in Figure 6e-h. As presented in Figure 6e-h, the surface creation parameters used for detecting rod diameters (green) help us collect data from every rod fitted for calculating the heights of the rods (semi-transparent yellow). To calculate the surface fitted rod's diameter, $d$, we average the reported length of these disk or donut shape fittings in the $\mathrm{X}\left(l_{x}\right)$ and $\mathrm{Y}\left(l_{y}\right)$ directions (see Methods) as, $d=\left(l_{x}+l_{y}\right) / 2$. As shown in Figure f-h, surface fitting analysis for evaluating the diameter of hydrophobic rods was done at high values in the z-direction and did not include any data from the short rods. Therefore, we did not apply any percentile to divide the data set into two and used the entire data set $\left(0^{\text {th }}-100^{\text {th }}\right.$ percentile $)$ while calculating the $d_{\text {avg }}$ of the hydrophobic (standard) rods. Finally, in Figure 6, we present rod height, $l_{\text {avg }}$ (Figure 6i), and diameter, $d_{a v g}$ (Figure 6j) calculated from the surfaces' 3D confocal and SEM images. Our results in Figure $6 \mathrm{j}$ demonstrates that the $d_{a v g}$ and $l_{a v g}$ of the rods calculated from SEM micrographs and 3D confocal images are in very good agreement. To prove this agreement statistically, we also performed a two-tail ANOVA test and realized no significant difference between the means of the reported $d_{a v g}$ and $l_{a v g}$ calculated from our SEM images and the confocal images. Such agreement between $d_{\text {avg }}$ and $l_{\text {avg }}$ results from our confocal imaging and SEM as a very established microscopic method shows us the application potential of our staining method to quantify the dimension of patterned surfaces with polysiloxane. However, when we focus on the deviations in the reported $d_{\text {avg }}$ and $l_{\text {avg, }}$ we realized that some of our staining strategies work better than the others. Compared to the data obtained from SEM images, the largest but non-significant difference was detected in the evaluated data from confocal images of the hydrophobic rods acquired after the ATTO-COOH containing droplet was removed from the surface (Figure 6d,h). Resolving the hydrophobic rods (especially their chimney-like shape) before removing the ATTO-COOH containing droplet from the surface is easier (Figure $5 \mathrm{~b}$ ) compared to the images taken after the droplet was removed from the surface 
(Figure 5c). Such a lack of resolution in Figure 5c impacts determining $d_{\text {avg }}$ and $l_{\text {avg }}$ results with more significant deviation than those from the SEM image analysis and other confocal images. However, it is essential to emphasize that we could collect statistically reasonable $d_{\text {avg }}$ and $l_{\text {avg }}$ from confocal images taken in different staining scenarios. As shown in the results in Figure 6, in general, our staining method successfully works (i) on different polysiloxane coated surfaces with different water repellency, (ii) after leaving sessile droplets with a different type of dyes, and (iii) even after the droplet was taken away after staining.

\section{Conclusion}

In summary, we introduced a new fluorescent imaging method to visualize the interface between sessile droplet and superhydrophobic or hydrophobic polysiloxane micro-and nanorod coated surfaces from a single rod resolution. With this new imaging method, we primarily acquired 2D, and 3D images from the interface between fluorescence dye-loaded sessile droplets and our surfaces. With the help of these images, for the first time, we visualized the presence of both micro-Cassie and nano-Cassie wetting states in our superhydrophobic surfaces, Cassie state micro-regions even long after $\mathrm{C} \rightarrow \mathrm{W}$ transition in our hydrophobic surfaces as well as changes in external and internal three-phase contact line shifting and pinning as a factor of time. These findings are essential to explain the time-dependent wetting kinetics of our coatings and other polymeric irregularly patterned (super) antiwetting surfaces. In the end, we used the $3 \mathrm{D}$ confocal images to quantify micro-and nano silicone rods' diameter and height, and we realized that these findings are in a statistically good agreement with the results calculated from the SEM micrographs.

For the theory of wetting, a mathematical description of the surface is essential. ${ }^{[12]}$ Therefore, previous studies were mainly focused on confocal imaging of the interface between sessile droplets (with dye) and fluorescently labeled pillars with regular size and regular center-tocenter pillar spacing. ${ }^{[9,12]}$ The use of such simple pillar geometry is essential to describe the wetting response of such patterned surfaces, for instance, their impalement dynamics. ${ }^{[8]}$ However, for more irregular surfaces as our polysiloxane patterns, there is no mathematical description available, and a theoretical description of wetting is out of reach. The presented confocal imaging method of our irregular surfaces in contact with sessile droplets provides, for the first time, means to obtain an empirical function in the form of $f(x, y)$ of the surface. Therefore, in terms of fundamental wetting phenomena, we believe the imaging method 
presented in this work could help the theory of wetting more irregular surfaces. In terms of application potential, besides quantifying the size of polysiloxane micro-and nano-rods, the ability to root-to-tip visualizing the rods even after the droplet removal can be applied in novel applications such as micro-stamping. With such micro-stamping, we can create micro-patterns on our polysiloxane patterns with different wetting or functionalization (e.g., hydrolysis). Such a new method of creating micro-functional patterns can be helpful in various emerging applications such as liquid patterning, miniaturized microfluidic separation, and diagnostics.

\section{Acknowledgements}

This work was financially supported by University of Zurich, Alfred-Werner-Legat and Swiss National Foundation (SNF). We acknowledge ZMB (University of Zurich) for providing access to their electron and confocal microscopy facilities. We thank Dr. Georg Artus for fruitful discussions and reviewing the manuscript, and Urs Zieger and Jana Döhner for their help in introducing the confocal microscopy techniques and helpful discussions. 


\section{Experimental Section}

Materials: Trichloroethylsilane (TCES, $\geq 97 \%$, Merck) was kept in a nitrogen-filled (waterfree) glove box during storing and handling. Atto633-Streptavidin $\left(M_{w} \text { is ca. } 55 \mathrm{kDa}\right)^{[23]}$ and Atto633-carboxyl $\left(M_{w}=652 \mathrm{~g} / \mathrm{mol}\right)$ fluorescent dyes were purchased from Atto-Tec (Siegen, Germany). They were dissolved in MiliQ water and then kept at $-20^{\circ} \mathrm{C}$.

\section{Chemical Vapor Deposition (CVD) of polysiloxane micro- \& nano-coatings.}

Cleaning and surface preparation of glass slides: Before coating, glass slides $(26 \mathrm{~mm} \times 76 \mathrm{~mm} \times$ $0.15 \mathrm{~mm}$, Menzel Gläser, Germany) were dipped inside of 10\% v/v Deconex (11 universal)/DI water solution and kept in US bath for 30 min for cleaning and surface activation (enhancing the surface hydrophilicity). After washing and drying (with steaming dry $\mathrm{N}_{2}$ ), the glass slides were fixed in a Teflon slide holder and placed inside a custom-built and sealed coating desiccator (ca. 6.5L inner volume). The desiccator has a gas inlet, a gas outlet, a septum, and a watch glass placed inside the desiccator to store the injected silane during coating. After placing slides in the desiccator, their surfaces were flushed for $1 \mathrm{~h}$ at a fixed relative humidity $(\mathrm{RH}, \%)$. Aimed RH was set by adjusting the flow (L/M) of the incoming wet and dry nitrogen while mixing inside a custom-built gas mixing chamber, which has exhaust access to the coating desiccator to set the RH inside. The gas mixing chamber's temperature was kept constant by circulating water around the gas mixing chamber with the help of an external thermostat $\left(23^{\circ} \mathrm{C}\right)$. The mixed gas's RH and temperature were monitored by a hygrometer (EE23, E+E Elektronik, Engerwitzdorf, Austria).

Superhydrophobic surface coating: After $1 \mathrm{~h}$ surface stabilization at $65 \%$ RH of 30 washed glass slides, the $\mathrm{N}_{2}$ gas flow is stopped by closing the inlet and outlet valves of the coating desiccator. Chemical vapor deposition ( $C D V$ ) for superhydrophobic coating was initiated after pipetting $1.5 \mathrm{~mL}$ TCES inside of the desiccator through the septum. CVD reaction was terminated after 16h. Before taking the coated substrates outside of the desiccator, we flushed the substrates for $10 \mathrm{~min}$ by only wet $\mathrm{N}_{2}(100 \% \mathrm{RH})$ and another $10 \mathrm{~min}$ by only dry $\mathrm{N}_{2}(0 \% \mathrm{RH})$. Stabilization, CVD coating, and final flushing were done at room temperature. The coated surfaces were finally rinsed under constant DI water flow before storing.

Hydrophobic surface coating: We flushed the surfaces of 5 washed glass slides by using 74\% RH for the hydrophobic coatings. Polymerization reaction took place while keeping the $74 \%$ 
RH (continuous flush of $\mathrm{N}_{2}$ ) running during the reaction period (16h) after the precursor addition $(392 \mu \mathrm{L}$ of TCES). Identical to the traditional way, after $16 \mathrm{~h}$ of polysiloxane growth, we flushed the surface first, $10 \mathrm{~min}$ by only dry $\mathrm{N}_{2}$ and then $10 \mathrm{~min}$ by only wet $\mathrm{N}_{2}$. Synthesis of the hydrophobic coating was entirely done under room temperature conditions. Also, after the coating, the surfaces were washed under the continuous flow of DI water. Since the coated surfaces are hydrophobic, remaining DI water droplets, after washing, were removed from the surface with the flush of a dry $\mathrm{N}_{2}$.

\section{Surface characterization and imaging of the coated surfaces.}

Static Contact angle $\left(\theta_{C A}\right)$ measurements: $\theta_{C A}$, droplet volume $\left(V / V_{i}\right)$, and droplet base diameter $\left(B D / B D_{i}\right)$ change as a factor of liquid/solid interaction time were quantified using a Krüss DSA100 Contact Angle device (Germany). $25 \mu \mathrm{L}$ of sessile droplets from MiliQ water or MiliQ water-based dye solutions were left on the coated surfaces using a Hamilton micro-syringe, and the static contact angles were determined after Young-Laplace fitting (Figure S3a,b). Each reported average value and standard deviations (sd) were calculated after at least 5 different $\theta_{C A}$ readings from the measurements on a minimum 3 different glass slides from the same CVD coating. All the measurements were done in lab conditions $\left(50 \% \mathrm{RH}, 23-24^{\circ} \mathrm{C}\right)$.

Scanning electron microscopy (SEM) imaging: For SEM imaging, the coated glass slides were broken into small pieces, and these pieces were placed on the top of double-sided carbon tape. Before the imaging, the samples were sputter-coated (Safematic CCU-010, Switzerland) with a $10 \mathrm{~nm}$ thick platinum layer. SEM micrographs were taken by using a Zeiss Supra 50VP electron microscopy (Zeiss, Germany). All the presented SEM images were taken by using $10 \mathrm{kV}$ of acceleration voltage and $30 \mu \mathrm{m}$ aperture size and after tilting the stage at $45^{\circ}$ or $90^{\circ}$. The length, diameter, and surface-to-surface distance of the coated polysiloxane rods were quantified by investigating SEM images using ImageJ image processing software. The measured length of rods in the $45^{\circ}$ tilted SEM images was divided by $\cos 45^{\circ}$ to calculate their real length. The diameter of the rods was measured at half of the maximum height. The diameter values were not divided by $\cos 45^{\circ}$. To eliminate the short rod data from the standard rod data in the hydrophobic coating, we used the cumulative distribution function to the histograms of the rods' calculated length data. Length values between the $0^{\text {th }}-45^{\text {th }}$ percentile were counted as short rods. The rest of the length data (between $45^{\text {th }}-100^{\text {th }}$ percentile) were counted as standard hydrophobic rods. 
Fluorescent staining of the (super)hydrophobic rods and their Confocal microscopy imaging: Identical to the CA measurements, all the Confocal microscopy imaging were performed in lab conditions $\left(50 \% \mathrm{RH}, 23-24^{\circ} \mathrm{C}\right)$. Before the confocal imaging, $25 \mu \mathrm{L}$ of a fluorescent dyecontaining water droplet $(50 \mu \mathrm{g} / \mathrm{mL})$ was left to rest on the surface. After a certain liquid/solid interaction time, we detected fluorescently labeled hydrophobic and superhydrophobic (twolevel topographic) silicone rods both in the 2D snapshot and z-stack 3D images acquired by operating a Leica SP5 Mid UV-VIS inverted confocal laser scanning microscopy (CLSM) equipped with a motorized stage $(x-y-z)$. A 64X oil (Type-G) immersed objective lens (HCX PL APO lambda blue 63x, NA=1.4, oil) and as two laser sources argon (visible, 20\% power) and $\mathrm{HeNe}$ lasers were used, and the pinhole kept at $95.5 \mu \mathrm{m}$ during imaging. Two detectors were used simultaneously to collect emissions from fluorescence molecules and reflection at the water/liquid interface. Lateral $\left(d_{x, y}\right)$ and axial $\left(d_{z}\right)$ resolution limits of our confocal configuration are calculated as $300 \mathrm{~nm}$ and $650 \mathrm{~nm}$, respectively. In general, Z-stack confocal imaging was done using a $0.04 \mu \mathrm{m}$ step size between each collected XY stacks.

Image (surface creation) analysis of the confocal images: 2D snapshot and 3D z-stack confocal images were processed using ImarisPro (Bitplane AG, Switzerland). First, we set displaying adjustments (max, min, and gamma $(\gamma)$ ) of each 2D or 3D image to optimize the histograms of the grey values of the detected signal (Table S1 and S2). The surface analysis feature ("add new surfaces" wizard) of ImarisPro was applied in our 3D z-stack images to quantify the length and diameter of fluorescently labeled micro- $\&$ nano-rods. After setting the display settings of the fluorescent signal (Table S2), we set the dimensions ( $\mathrm{x}, \mathrm{y}, \mathrm{z}$ in length, $\mu \mathrm{m}$ ) of the volume-ofinterest to run the surface fitting analysis (Table S2). In general, XY-orthoslicer (in the zdirection) showing a uniform reflectance signal (red color) and eventually the exact point where the root of the rods starts appearing, set as the bottom limit in the z-direction of such volume of interest (see Figure S6, S9, and S10c). For the highest point of such volume of interest, we set the highest possible z-distance (limits of the acquired z-stack image). Subsequently, in the "add new surfaces" wizard, we entered the following parameters (and their units, meanings) in a sequence: Surface Details ( $\mu \mathrm{m}$, alter the smoothing of the final surface fitting), Background Subtraction ( $\mu \mathrm{m}$, largest sphere diameter to fit), Threshold (\%, used for defining the border between included and excluded grey values) and Quality (\%, used to quantify the detected signal quality to accept for analysis). All the detected features with a dimension smaller than $0.1 \mu \mathrm{m}$ were eliminated. For surface fitting analysis of length and diameter of rods in a particular z-stack image, we used identical display adjustment and surface creating parameters. However, 
to generate disc shape (or donut shape) surface creation to quantify the rods' diameter, we entered a shorter distance in z-dimension of the volume-of-interest (Figure 5c-d). Disc shape surfaces were created close to the middle of the rod length, and the rod diameter of each rod is calculated by taking the mean of the $\mathrm{X}$ and $\mathrm{Y}$-direction distance of the disk fittings $\left(\left(l_{X}+l_{y}\right) / 2\right)$. All the display settings, volume-of-interests information, and the surface creation parameters used in this work are listed in Table S2. After collecting the length and diameter data from the surface creation analysis, similar to SEM image analysis, we fitted CDF to the average length of short and standard hydrophobic rods histogram of the length data. Length values in the histograms within the $0^{\text {th }}$ and $45^{\text {th }}$ percentile and $45^{\text {th }}$ and $100^{\text {th }}$ percentile were used to calculate the short and standard rods' average length, respectively. Since the entire surface fitting of hydrophobic rods to evaluate their diameters were done at relatively high (zdirection) volume-of-interest from the rods' roots, we could avoid collecting data from the short rods (Figure 5f-h). Thus, the entire diameter dataset from hydrophobic rods was used to calculate the average diameter of the standard (long) rods. 


\section{References}

[1] M. Liu, S. Wang, L. Jiang, Nat. Rev. Mater. 2017, 2, 1.

[2] T. Nishino, M. Meguro, K. Nakamae, M. Matsushita, Y. Ueda, Langmuir 1999, 15, 4321.

[3] A. Tuteja, W. Choi, M. Ma, J. M. Mabry, S. A. Mazzella, G. C. Rutledge, G. H. McKinley, R. E. Cohen, Science, 2007, 318, 1618.

[4] Z. Chu, S. Seeger, Chem. Soc. Rev. 2014, 43, 2784.

[5] J. Zimmermann, G. R. J. Artus, S. Seeger, J. Adhes. Sci. Technol. 2008, 22, 251.

[6] N. J. Shirtcliffe, G. McHale, M. I. Newton, C. C. Perry, F. B. Pyatt, Appl. Phys. Lett. 2006, 89, 104106.

[7] C. H. Kung, P. K. Sow, B. Zahiri, W. Mérida, Adv. Mater. Interfaces 2019, 6, 1900839.

[8] P. Papadopoulos, L. Mammen, X. Deng, D. Vollmer, H.-J. Butt, Proc. Natl. Acad. Sci. 2013, 110, 3254 .

[9] P. Papadopoulos, X. Deng, L. Mammen, D.-M. Drotlef, G. Battagliarin, C. Li, K. Müllen, K. Landfester, A. Del Campo, H.-J. Rgen Butt, D. Vollmer, Langmuir 2020, 28,8392 .

[10] K. M. Smyth, A. T. Paxson, H.-M. Kwon, K. K. Varanasi, Surf. Innov. 2013, 1, 84.

[11] D. Daniel, J. V. I. Timonen, R. Li, S. J. Velling, M. J. Kreder, A. Tetreault, J. Aizenberg, Phys. Rev. Lett. 2018, 120, 244503.

[12] F. Schellenberger, N. Encinas, D. Vollmer, H. J. Butt, Phys. Rev. Lett. 2016, 116, 2.

[13] Y. Y. Quan, Z. Chen, Y. Lai, Z. S. Huang, H. Li, Mater. Chem. Front. 2021, 5, 1655.

[14] J. Zimmermann, M. Rabe, D. Verdes, S. Seeger, Langmuir 2008, 24, 1053.

[15] G. R. J. Artus, S. Jung, J. Zimmermann, H.-P. Gautschi, K. Marquardt, S. Seeger, Adv. Mater. 2006, 18, 2758.

[16] J. Zimmermann, S. Seeger, G. Artus, S. Jung, Superhydrophobic Coating - WO2004 $113456,2004$.

[17] G. R. J. Artus, S. Olveira, D. Patra, S. Seeger, Macromol. Rapid Commun. 2017, 38, 1.

[18] T. Verho, J. T. Korhonen, L. Sainiemi, V. Jokinen, C. Bower, K. Franze, S. Franssila, P. Andrew, O. Ikkala, R. H. A. Ras, Proc. Natl. Acad. Sci. U. S. A. 2012, 109, 10210.

[19] D. Mampallil, H. B. Eral, Adv. Colloid Interface Sci. 2018, 252, 38.

[20] R. Blossey, A. Bosio, Langmuir 2002, 18, 2952.

[21] V. Dugas, J. Broutin, E. Souteyrand, Langmuir 2005, 21, 9130.

[22] A. Stojanovic, S. Olveira, M. Fischer, S. Seeger, Chem. Mater. 2013, 25, 2787.

[23] M. Howarth, A. Y. Ting, Nat. Protoc. 2008, 3, 534. 
[24] A. Lafuma, D. Quéré, Nat. Mater. 2003, 2, 457.

[25] F. Y. H. Lin, D. Li, Chem. Eng. Sci. 1995, 50, 2633.

[26] D. Li, F. Y. H. Lin, A. W. Neumann, J. Colloid Interface Sci. 1991, 142, 224.

[27] J. L. Garcia-Cordero, Z. H. Fan, Lab Chip 2017, 17, 2150. 